\title{
TerraSAR-X In-Orbit Antenna Model Verification Results
}

\author{
Markus Bachmann ${ }^{1}$, Marco Schwerdt ${ }^{1}$, Benjamin Bräutigam ${ }^{1}$ \\ ${ }^{1}$ German Aerospace Center (DLR), Oberpfaffenhofen, 82234 Weßling, Germany, \\ Tel: 08153 28-2395, Fax: 08153 28-1449 \\ markus.bachmann@dlr.de
}

\begin{abstract}
TerraSAR-X is a highly flexible X-band radar satellite. Its primary objective is the acquisition of high quality SAR images in a multitude of possible acquisition modes. The great amount of antenna patterns needed for image acquistion requires an antenna model accurately describing all beams. To guarantee the required image quality, the model is verified in-orbit. As TerraSAR-X is scheduled for launch in June 2007, this paper will present the necessity of the antenna model and the methods to verify it.
\end{abstract}

\section{Motivation}

TerraSAR-X is a versatile X-Band SAR satellite built in a Public Private Partnership between DLR and Astrium. The main payload of TerraSAR-X is a synthetic aperture radar instrument to acquire high quality radar images of the earth's surface.

\section{A. Instrument specification}

The radar instrument comprises an active phased array antenna which allows flexible beam forming. The antenna characteristics can be accurately modelled by mathematical equations based on on-ground measurements. The antenna with its $4.8 \mathrm{~m}$ length and $0.7 \mathrm{~m}$ width consists of 384 slotted wave-guides arranged in 12 panels in azimuth direction (columns) each with 32 subarrays (rows) [1], [3]. The nominal antenna pointing is $33.8^{\circ}$ away from nadir. Right and left looking acquisition is realised by satellite roll manoeuvres.

The individual subarrays are driven by Transmit-Receive Modules (TRMs) adjustable in amplitude and phase by applying complex excitation coefficients. This enables to steer and form the beam in azimuth and elevation direction. For the multitude of standard acquisition modes - like nominal Stripmap, ScanSAR or Spotlight - and the several experimental modes - like quad-pol mode, wide band operation or along-track interferometry - more than 10000 different beams can be commanded.

\section{B. Antenna model necessity}

This great amount of beams is one highlight but also a challenge of the whole mission and the main driver for the need of an accurate antenna model.

The model is primarily used to generate the antenna patterns needed by the processing system to correct for the antenna characteristic of each beam. The characteristic is mapped in the acquired SAR images i. e. the pattern is visible in the pixel intensity as the signal reflection of the ground is added up with the antenna pattern in range. In azimuth, the pattern is contained in the Doppler spectrum and hence needed for correct Doppler estimation.

To ensure the challenging radiometric accuracy requirement of TerraSAR-X, an accurate calibration of the antenna patterns has to be performed. In former missions like ENVISAT/ASAR [10] all different beams were calibrated separately by measuring their patterns in-orbit. This solution is not applicable to the multitude of beams of TerraSAR-X. Therefore, a new calibration approach will be realised. Instead of calibration all beams individually, the antenna model will be verified first to generate all beam patterns.

A second important reason for the antenna model is the optimisation of the beam excitation coefficients prior to launch to achieve an optimum performance for the full performance beams in terms of Noise Equivalent Sigma Zero (NESZ) and Total Ambiguity Ratio (TAR).

\section{ANTENNA MODEL THEORY}

The antenna model calculates radiation patterns by superposition of measured embedded subarray patterns weighted by beam excitation coefficients considering the exact geometrical dimensions of the antenna.

For cuts in elevation and azimuth the radiated pattern $F_{\text {Beam }}$ is calculated by [2]

$$
\begin{aligned}
\vec{F}_{\text {Beam }}(\varepsilon, \alpha)=\sum_{m=0}^{M-1} \sum_{n=0}^{N-1}\left(\vec{C}_{S A, m n}(\varepsilon, \alpha) \cdot a_{m n} \cdot E_{S A, m n}\right. \\
\left.e^{j k \sin \varepsilon \cos \alpha\left(-\frac{N-1}{2}+n\right) \Delta y} \cdot e^{j k \cos \varepsilon \sin \alpha\left(-\frac{M-1}{2}+m\right) \Delta x}\right)
\end{aligned}
$$

with the desired elevation and azimuth angle $\varepsilon$ and $\alpha$, the amount of subarrays in $N$ rows and $M$ columns, the intersubarray distances $\Delta x$ and $\Delta y$ and the wavenumber $k$.

The embedded subarray patterns $C_{S A}$ describe the radiation characteristics of the individual subarray elements embedded into the whole antenna and have to be given for each row, column, elevation and azimuth angle. The commanded excitation coefficients are given by $a$. Finally, the error matrix $E_{S A}$ describes drifting or failed antenna elements which are determined via internal calibration loops and orthogonal code sequences applied to the TRMs [3][4]. 
The TerraSAR-X operation and calibration approach requires a very high accuracy of the antenna model better than $0.2 \mathrm{~dB}$ of pattern shape and absolute gain. Hence, the antenna model has to reproduce the behaviour of the real antenna as well as possible. Therefore, accurately on ground measured characterisation data i. e. embedded subarray patterns are used.

\section{IN-ORBIT VERIFICATION}

\section{A. Verification method}

The antenna model was validated on-ground by comparing modelled patterns with measured patterns. This validation was performed successfully showing the excellent performance of the antenna model [5]. To verify this antenna model performance for the whole antenna in space, in-orbit measurements will be performed during the commissioning phase in the first weeks after launch.

The verification is performed with really measured beams and can be divided into three main tasks:

- measurements across the rainforest to verify the elevation pattern shape

- ScanSAR measurements over rainforest to verify the calculated vs. simulated peak-to-peak gain variation, and

- the use of ground receivers to verify the azimuth pattern.

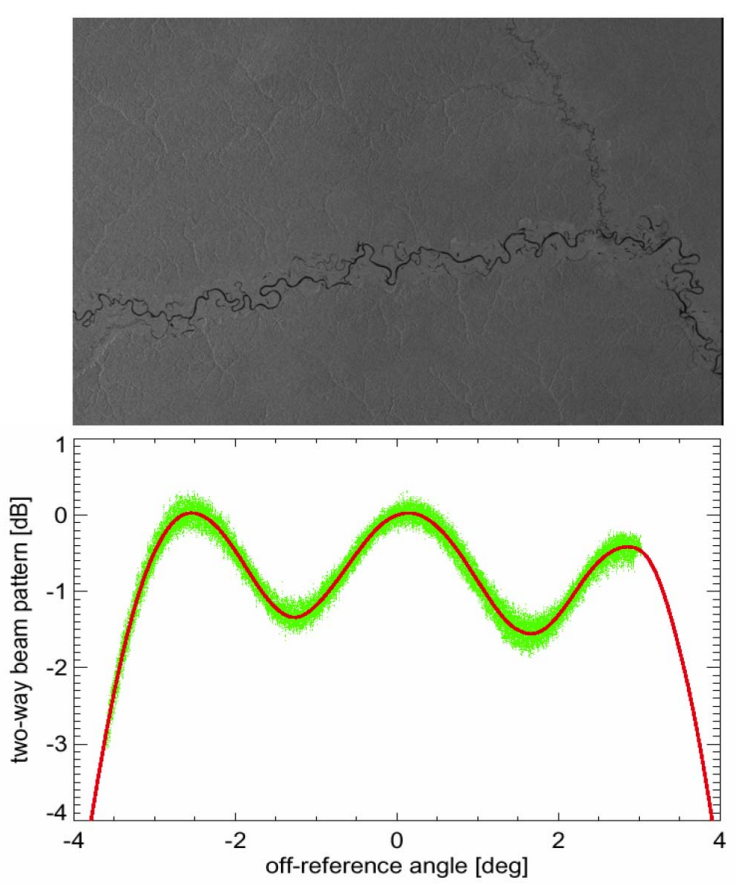

Fig. 1 ASAR elevation pattern (red line) derived from the range profile (green points) of the above rain forest scene

For elevation, the simulated relative antenna patterns are compared with estimated patterns from SAR data acquired over Amazon rainforest. As Amazon rainforest is a quite homogeneous scatterer, the pattern shape is clearly visible in the SAR raw data. However, as this raw data are pulses without any processing, the processed image is used for pattern estimation. In the nominal processing chain, the accurate geometrical position of the data is determined and annotated after azimuth and range compression. Then the patterns mapped in the image data is corrected with the available modelled reference patterns [6].

For pattern estimation, this reference pattern correction is reversed. Then, the image is freed of disturbing image areas like rivers or de-forested areas and all range lines are summed up to obtain the so called gamma profile. This is depicted in the lower part of Fig. 1. The light, green noise like ripple is the gamma profile, the smooth line a fit which now can be compared to the modelled reference pattern.

The peak-to-peak gain prediction i. e. the prediction of the variation of the gain in the maximum relative from beam to beam in elevation is verified evaluating ScanSAR data. In ScanSAR acquisitions, the beam is switched sequentially from burst to burst between four neighbouring beams to get a broader swath width than for normal Stripmap acquisitions. In Level1B-Products, the four swaths are still available in separate images. By generating the un-normalised gamma profile for each of the four swaths, the relative gain deviation can be determined.

The verification of the antenna pattern in azimuth direction will be performed for the transmit pattern using the DLR ground calibration [7] equipment in form of ground receivers. These receivers measure the amplitude of the pulses transmitted by the TerraSAR-X satellite over time, see the ENVISAT/ASAR example in Fig. 2. Transformed to the antenna elevation angles and corrected by position information, the azimuth antenna pattern is obtained and can be compared to the modelled pattern.

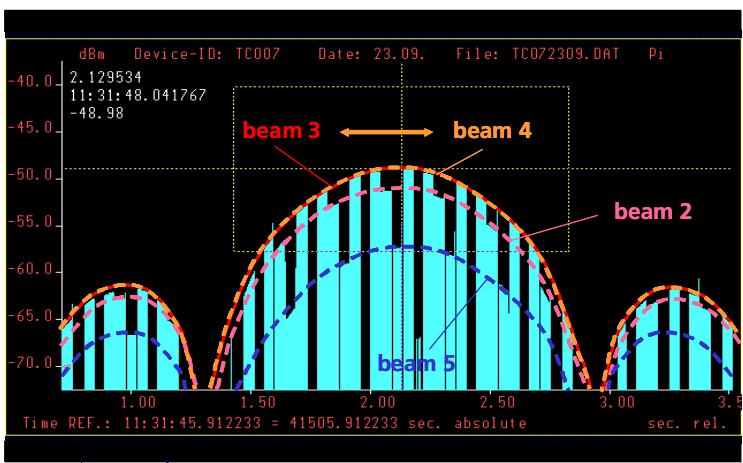

Fig. 2 Transmit pulses of the ASAR/ENVISAT instrument as function of time recorded by a deployed ground receiver. The reduced amplitudes show the switching of the instrument from beam to beam and the corresponding envelopes represents the one-way (transmit) azimuth antenna patterns of ASAR in ScanSAR operation

\section{B. Support of the External Calibration}

After its verification, the antenna model will also help to achieve a short commissioning phase [8]. Both the pointing 
calibration and the determination of the absolute calibration factor will be supported by the antenna model.

For pointing calibration, the exact pointing of the antenna will be measured. In elevation, this will be performed over Amazon rainforest again. In azimuth, the deviation of the geometrical to the measured Doppler will be evaluated using the SAR processor. For comparison, ground receivers will be used, combining the in-orbit time and position of the satellite with the azimuth position and time at the received patterns.

Assuming a properly working antenna model, the absolute calibration factor $i$. e. the equivalent radar cross section of targets does not have to be measured for all relevant beams. It will be determined for several few beams only. Taking the processor gain into account and assuming properly applied absolute antenna patterns correction in the SAR processor, the absolute calibration factor is assumed to be equal on all beams.

By this approach it is possible to shorten the time and the effort for the absolute radiometric calibration during the commissioning phase as the absolute calibration factor of only a few beams has to be measured.

\section{SOFTWARE TOOLS}

Several software tools have been developed or extended both for the in-orbit verification of the model as well as for optimisation and generation of the antenna pattern via the antenna model.

\section{A. Antenna pattern analysis}

As described earlier in chapter III.A, the verification for azimuth and elevation will be performed by two different approaches.

The tool for elevation pattern estimation reads a TerraSAR-X SAR image. It applies certain back-correction to the image to get rid of the influences of the calibration correction applied in the processor and sums the range lines to achieve a gamma profile. By combining gamma profiles of several passes and scenes, a quite good pattern is achieved. This is then compared to the modelled pattern.

For azimuth verification, the data read via ground receivers is evaluated. By incorporating the exact position and alignment of the transponder, the orbit vector of the satellite and considering the transponder antenna patterns, the azimuth pattern is determined. Combining the azimuth pattern of several over-flights and transponders, the modelled pattern can again be compared to the measured data.

\section{B. Excitation coefficient optimisation}

The Antenna Excitation Generator Software uses the antenna model to generate antenna settings for optimal instrument performance of TerraSAR-X. It is important to achieve high sensitivity over a large angular range $\left(20^{\circ}-45^{\circ}\right.$ antenna look angle) as well as good suppression of ambiguities. The optimisation process is based on different quality parameters like two-way antenna gain, ripple, and ambiguity ratio which themselves have their basis in different performance estimations. The estimation is performed by varying the antenna excitation coefficients, generating the antenna pattern each time, comparing the actual pattern against the given quality parameters and the best pattern up to that time. A launch set of optimised excitation coefficients is generated prior to the mission and will be adapted during mission time through re-optimisation in case of phase drifts or amplitude degradation in the TRMs.

An example for the optimisation is shown in Fig. 3. The thin black curve is the optimised pattern without errors. Assuming severe phase drifts and amplitude degradation in the TRMs, the pattern degrades to the brown thin curve. After re-optimisation, the requirements in terms of mask and gain (straight green/blue lines) are met again, shown by the red thick curve.

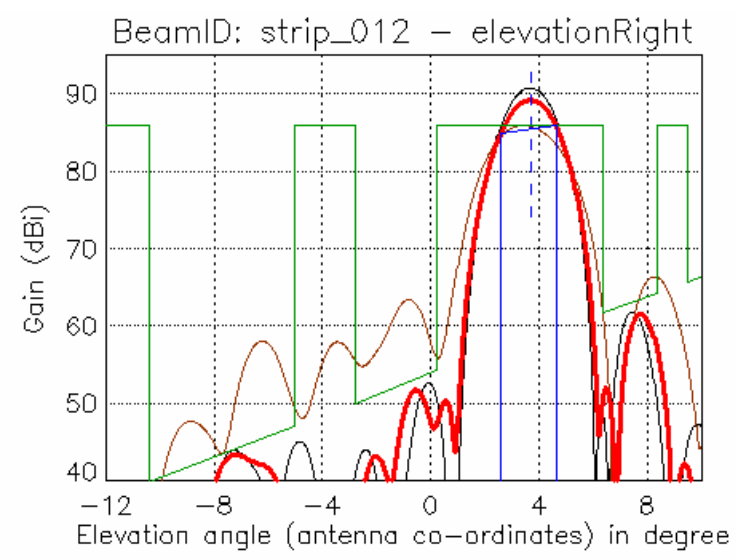

Fig. 3 Results of the antenna pattern optimisation

\section{Reference pattern generation}

In addition to the optimisation, antenna reference patterns (Fig. 4) are calculated for each mode, beam and polarisation using the optimised excitation coefficients. These reference patterns are needed by the SAR processor to eliminate the pattern shape from the SAR images (relative radiometric calibration). Here, the accuracy of the antenna model and the resulting reference patterns is important as even small deviations of some tenths of $\mathrm{dBs}$ are visible in the merging areas of ScanSAR images, where four neighbouring swaths are merged into one image.

Finally, the antenna patterns are used by the performance estimation [9] prior to launch and during flight to estimate and monitor the actual TerraSAR-X performance. 


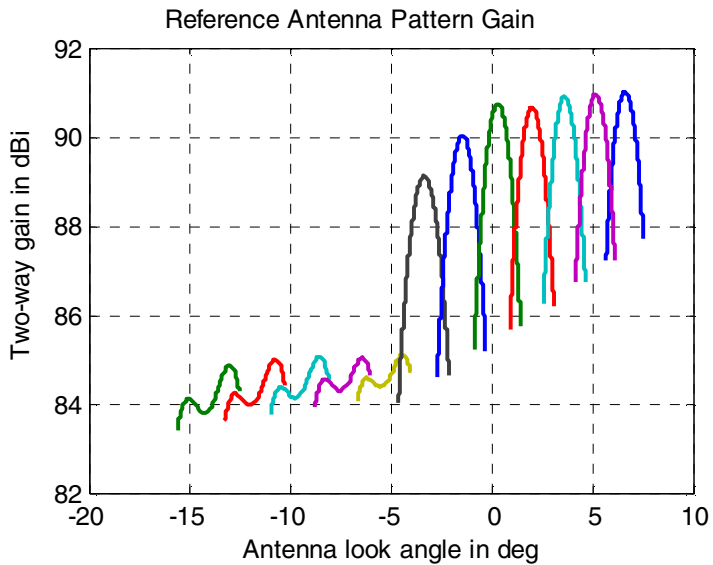

Fig. 4 Results of the pattern optimisation for the TerraSAR-X full performance beams

\section{SUMMARY}

The TerraSAR-X antenna model is utilised for generating the reference antenna patterns for processing and for beam optimisation. To ensure the specified product quality, its accuracy has to be verified in orbit during the commissioning phase following the TerraSAR-X launch scheduled for June 2007. The methods for calibrating the antenna model and supporting the calibration in general are published here, as they are of interest to both the scientific community and commercial users of TerraSAR-X products. Due to the TerraSAR-X launch delay, the antenna model is not verified yet. However, the presentation of this paper on EuRAD will contain the first results of this antenna model verification.

\section{REFERENCES}

[1] R. Werninghaus, W. Balzer, S. Buckreuss, J. Mittermayer, P. Mühlbauer, W. Pitz, "The TerraSAR-X Mission," Proceedings of EUSAR 2004, Ulm, Germany, May 25-27, 2004.

[2] B. Grafmüller, A. Herschlein, C. Fischer: The TerraSAR-X Antenna System; Radar Conference, 2005 International, May 2005

[3] D. Hounam, M. Schwerdt, and M. Zink, "Active Antenna Module Characterisation by Pseudo-Noise Gating," in 25th ESA Antenna Workshop on Satellite Antenna Technology, Noordwijk, Netherl., 2002.

[4] B. Bräutigam, M. Schwerdt, M. Bachmann, M. Stangl: "Individual T/R Module Characterisation of the TerraSAR-X Active Phased Array Antenna by Calibration Pulse Sequences with Orthogonal Code, $26^{\text {th }}$ International Geoscience And Remote Sensing Symposium, Barcelona, Spain, 2007

[5] M. Bachmann, M. Schwerdt, B. Bräutigam, B. Grafmüller, A Herschlein, J. L. Álvarez-Pérez: The TerraSAR-X Antenna Model Approach; To be published in Proceedings on INICA 07, Munich, March 2007

[6] J. L. Álvarez-Pérez, M. Schwerdt, M. Bachmann: TerraSAR-X Antenna Pattern Estimation by Complex Treatment of Rain Forest Measurements; Geoscience and Remote Sensing Symposium, IGARSS 2006, Denver, USA, July 2006

[7] B. Bräutigam, M. Schwerdt, M. Bachmann, B. Döring: Results from TerraSAR-X Geometric and Radiometric Calibration,

[8] B. Bräutigam, M. Schwerdt, M. Bachmann: The External Calibration of TerraSAR-X, a Multiple Mode SAR-System; Proceedings of European Conference on Synthetic Aperture Radar (EUSAR), Dresden, Germany, May 2006

[9] J. Márquez-Martinez, C. Gonzalez, J. Mittermayer: TerraSAR-X Performance Update; Proceedings of European Conference on Synthetic Aperture Radar (EUSAR), Dresden, May 2006

[10] M. Zink, B. Rosich: Antenna Elevation Pattern Estimation from Rain Forest Acquisitions, ENVISAT/ASAR Calibration Review(ECR) of ESTEC European Space Agency (ESA), Noordwijk, Netherland, 2002 